Check for updates

Cite this: Phys. Chem. Chem. Phys., 2021, 23, 13878

Received 10th March 2021, Accepted 4th June 2021

DOI: $10.1039 / d 1 c p 01082 d$

rsc.li/pccp

\title{
Tin deposition on ruthenium and its influence on blistering in multi-layer mirrors $\dagger$
}

\author{
Chidozie Onwudinanti, (D) *ab Geert Brocks, ${ }^{\text {bcd }}$ Vianney Koelman, ${ }^{\text {abc }}$ \\ Thomas Morgan ${ }^{a}$ and Shuxia Tao (D)*bc
}

\begin{abstract}
An atomistic description of tin deposition on ruthenium and its effect on blistering damage is of great interest in extreme ultraviolet (EUV) lithography. In EUV machines, tin debris from the EUV-emitting tin plasma may be deposited on the mirrors in the optical path. Tin facilitates the formation of hydrogenfilled blisters under the ruthenium top layer of the multi-layer mirrors. We have used Density Functional Theory (DFT) to show that tin deposition on a clean ruthenium surface exhibits a film-plus-islands (Stranski-Krastanov) growth mode, with the first atomic layer bonding strongly to the substrate. We find that a single tin layer allows hydrogen to reach the ruthenium surface and subsurface more easily than on clean ruthenium, but hydrogen penetration through the tin film becomes progressively more difficult when more layers are added. The results indicate that hydrogen penetration and blistering occur when only a thin layer of tin is present.
\end{abstract}

\section{Introduction}

The undesirable formation of blisters, pockets of hydrogen gas under the top layer of mirrors in extreme ultraviolet (EUV) lithography machines, is a determining factor in the operational lifetime of these mirrors. Blistering causes a loss of reflectivity, the single most important property of the multi-layer mirrors (MLMs). This presents an obstacle to achieving high-volume manufacturing of next-generation integrated circuits.

The formation of blisters in EUV mirrors is a tale of three elements: ruthenium, hydrogen and tin. Ruthenium is used as the top layer in a stack of 40-60 molybdenum/silicon bilayers, each about 6 to 7 nanometres thick. ${ }^{1,2}$ Hydrogen is present, at near-vacuum pressure, to serve as a protective buffer for the surface and also to etch off impurities such as tin, carbon, and oxygen. ${ }^{3-5}$ Tin is the source of EUV light in the machines, as tin droplets are excited by a laser to generate EUV-emitting tin plasma. Tin debris can then be deposited on other surfaces in the machine, including those of the multilayer collector and mirrors in the optical path. Hydrogen comes into contact with the ruthenium cap, and may penetrate the surface, diffuse

\footnotetext{
${ }^{a}$ DIFFER-Dutch Institute for Fundamental Energy Research, Eindhoven The Netherlands

${ }^{b}$ Center for Computational Energy Research, Eindhoven, The Netherlands ${ }^{c}$ Department of Applied Physics, Eindhoven University of Technology, Eindhoven, The Netherlands. E-mail: s.x.tao@tue.nl

${ }^{d}$ Computational Materials Science, Faculty of Science and Technology and MESA+ Institute for Nanotechnology, University of Twente, Enschede, The Netherlands $\dagger$ Electronic supplementary information (ESI) available. See DOI: 10.1039/ d1cp01082d
}

through the bulk to the interface(s) of the multi-layer structure, and recombine to form pockets of $\mathrm{H}_{2}$ gas. When these pockets reach a critical pressure, the layers separate, resulting in blisters and loss of reflectivity.

Our study of tin deposition on ruthenium and its effect on blistering is motivated by the observation that blistering proceeds more rapidly in the presence of tin debris. In previous publications, we examined the effect of tin on hydrogen penetration into ruthenium, and the mechanism via which hydrogen becomes trapped under the normally near-impermeable ruthenium. ${ }^{8,9}$ We found that the proximity of tin to a hydrogen atom on the surface resulted in a lowering of the energy barrier to its penetration into the subsurface; furthermore, we showed a that the saturation of the surface with hydrogen and tin inhibited diffusion of hydrogen out of the ruthenium layer from the top surface. These computational studies of the effect of tin have so far been limited to the interactions of ruthenium and hydrogen with dispersed tin atoms and tin hydrides on the exposed ruthenium surface.

Tin has long been used as a protective coating for metals, and thin films have been reported to hinder hydrogen permeation into iron. ${ }^{10}$ Tin deposition on ruthenium has been studied in experiment, exploring the effect of deposition temperature on the structure of deposited tin, ${ }^{11}$ and its surface alloying with lead; ${ }^{12} a b$ initio studies have been reported for sub-monolayer tin films on ruthenium. ${ }^{13}$ For the EUV application the interaction with atomic hydrogen is paramount, and this has been a topic of intense study for the past decade. These experiments focus primarily on the efficiency of Sn removal. Studies of tin etching from representative MLM 
samples show that both the rate of Sn removal and blistering of samples depend strongly on the nature of the top layer and the surface morphology of the tin layer; in these experiments, tin layers of up to $20 \mathrm{~nm}$ were etched from diverse materials. ${ }^{14-16}$ To the best of our knowledge, no study of the atomistic interaction of hydrogen with multi-layer tin on ruthenium has been reported. Our aim, therefore, is to examine how the presence of multiple layers affects the likelihood of hydrogen permeation and blistering.

In this study, we look at the energetics of tin growth on a ruthenium surface, and examine the paths of hydrogen through the tin on the ruthenium surface. Our calculations show that tin binds strongly to the clean ruthenium surface, with the energies indicating a film-plus-islands growth mode (the so-called Stranski-Krastanov mode). The monolayer of tin changes the energy landscape in such a manner that when the $\mathrm{Sn} / \mathrm{Ru}$ surface is exposed to atomic hydrogen, the gas passes into the ruthenium subsurface relatively easily, with multiple layers of tin becoming progressively less permeable.

The article is structured thus: we report first the calculated structure for one densely-packed layer of tin on clean and hydrogen-covered ruthenium surfaces. Thereafter we study the evolution of the structures and energies of layers of tin. This is followed by a look at the interaction of hydrogen with the tin-on-ruthenium system. Finally we perform transition state calculations for hydrogen diffusion through the tin overlayer(s), and discuss the implications of the computed energies for blistering in EUV mirrors.

\section{Computational methods}

The calculations in this article employ DFT as implemented in the Vienna $a b$ initio Simulation Package (VASP). ${ }^{17-19}$ We follow the generalized gradient approach proposed by Perdew, Burke, and Ernzerhof $(\mathrm{PBE}),{ }^{20}$ with the following convergence criteria: a kinetic energy cutoff of $400 \mathrm{eV}$, a residual force criterion between $1 \times 10^{-2} \mathrm{eV}^{-1}$ and $5 \times 10^{-2} \mathrm{eV}^{-1}$, with $1 \times 10^{-5} \mathrm{eV}$ as the energy convergence criterion. Calculations for reference energies of bulk $\mathrm{Sn}$ and Ru were performed in a $2 \times 2 \times 2$ unit cell, while slab calculations were done with a rectangular unit cell, as described in Section 3.1. Bulk calculations were done with a $(9 \times 9 \times 9) \Gamma$-centred $k$-point grid, while slab calculations were performed with a $(9 \times 9 \times 1) \Gamma$-centred $k$-point grid with at least the top 2 layers of the slabs allowed to relax in the optimisation. Transition state calculations were carried out using the Climbing Image Nudged Elastic Band (CINEB) algorithm, ${ }^{21}$ with a force criterion between $1 \times 10^{-2}$ and $3 \times$ $10^{-2} \mathrm{eV} \AA^{-1}$ and one (1) to seven (7) intermediate geometries for the transition state search.

The calculated lattice parameters for hexagonal closepacked (hcp) ruthenium are $a=2.71 \AA$ and $c / a=1.585$, which are in good agreement with experimental results, $2.71 \AA$ and 1.58 , respectively. ${ }^{22}$ The $\mathrm{Ru}(0001)$ surface is modelled as a slab of seven (7) layers using a $(\sqrt{3} \times 3)$ rectangular unit cell, with approximately $15 \AA$ of vacuum between the periodic images in the $z$-direction.

The energy of adsorption is computed per the definition

$$
E_{\mathrm{ads}}=\frac{1}{n}\left(E_{\mathrm{nA}, \mathrm{slab}}-E_{\mathrm{slab}}-n E_{\text {Aref }}\right),
$$

where $E_{\mathrm{nA}, \mathrm{slab}}, E_{\mathrm{slab}}$, and $E_{\text {Aref }}$ stand respectively for the total energies of the slab with $n$ adsorbed atoms of element $\mathrm{A}$, the slab without the adsorbed atom(s), and the reference energy of the adsorbed atom(s). This reference energy is half the energy of the $\mathrm{H}_{2}$ molecule for hydrogen, and the energy of one $\beta$-tin atom for adsorbed tin.

Due to the very low mass of the hydrogen atom, its diffusion is, in general, influenced by zero-point energy (ZPE). Therefore the energy barriers reported in the subsequent chapters include ZPE corrections. The ZPE is calculated by the relation

$$
\mathrm{ZPE}=\frac{1}{2} \sum_{i} h v_{i},
$$

where $v_{i}$ is a real normal mode frequency. For example, the zero point energy for a hydrogen molecule $\left(\mathrm{H}_{2}\right)$ calculated thus is $0.27 \mathrm{eV}(0.135 \mathrm{eV}$ per $\mathrm{H}$ atom), corresponding to a vibrational mode of $4354 \mathrm{~cm}^{-1}$, in good agreement with the experimentally-determined value of $4401 \mathrm{~cm}^{-1} \cdot{ }^{23}$

\section{Results and discussion}

\subsection{Structure and energies of $\mathrm{Sn}$ on clean $\mathrm{Ru}$}

Tin deposition on the $\mathrm{Ru}(0001)$ surface has been modelled in a series of DFT calculations. As the ruthenium and tin differ significantly both in crystal structure and atomic radius, an appropriate unit cell for the calculation must be chosen to minimise the mismatch. The rectangular unit cell (shown in Fig. 1a) successfully accommodates the structure of the tin overlayer, which is not necessarily hexagonal (see ESI $\dagger$ for a comparison of Sn slabs in the rectangular unit cell and $\beta$-Sn lattice). The chosen reference state of tin is $\beta$-Sn shown in Fig. 1d, the so-called white tin, which is stable at room temperature, close to the operating conditions of EUV machines. Although $\alpha$-Sn is most stable at low temperatures, the choice of reference state has no consequence for the calculations or conclusions.

We have carried out geometry optimisations for varying numbers of complete layers of $\mathrm{Sn}$ on the Ru surface. We first determine the structure of the single atomic layer of $\mathrm{Sn}$, and subsequently find the lowest-energy configurations for each newly-added layer. We report the adsorption energies for the successive layers, and discuss the implications for the growth of tin on ruthenium.

1 layer. Due to the large size of the tin atom, the most dense packing of a tin layer on the Ru surface has $2 \mathrm{Sn}$ atoms per $3 \mathrm{Ru}$ atoms (giving $4 \mathrm{Sn}$ atoms in the unit cell), which we designate 2/3ML, shown in Fig. 1. To find the structure of this layer, we performed first a geometrical optimisation of a 7-layer $\mathrm{Ru}(0001)$ slab, then a further optimisation of the resulting slab with the $\mathrm{Sn}$ atoms on the surface, with all atoms permitted to relax. 


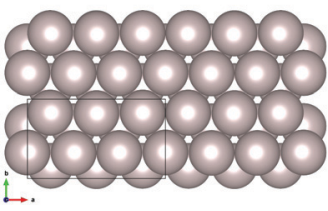

(a)

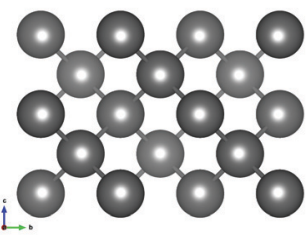

(c)

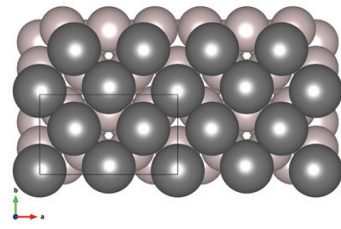

(b)

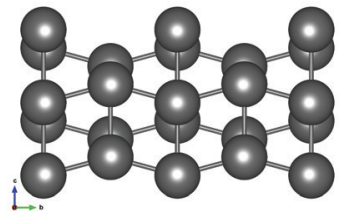

(d)
Fig. 1 (a) Top view of Ru(0001) slab (black rectangle shows unit cell); (b) structure of $2 / 3 \mathrm{ML}$ Sn on Ru; (c) $\alpha$-Sn crystal; (d) $\beta$-Sn. Dark spheres represent $\mathrm{Sn}$, light spheres $\mathrm{Ru}$.

The result of this calculation, repeated with various starting positions of the Sn atoms, is the structure of the single Sn layer, illustrated in Fig. 1.

This coincides with the $(\sqrt{3} \times 3)$-rect structure reported from experiment ${ }^{11,12}$ and first principles. ${ }^{13}$ The atoms in the first layer show a large negative adsorption energy of $-1.19 \mathrm{eV}$, per eqn (1), with the energy of one $\beta$-Sn atom as the reference. For projected density of states of the $\mathrm{Sn} / \mathrm{Ru}$ interface, see Supporting Information. The strong bond between the Sn and $\mathrm{Ru}$ atoms is attested by the difficulty of etching Sn completely from $\mathrm{Ru}^{14}$

2 layers. Several calculations were performed to ascertain the preferred structure of 2 layers of Sn on Ru. Varying configurations of 4 additional $\mathrm{Sn}$ atoms were placed on the single-layer structure of Fig. 1, and the resulting systems were allowed to relax in a standard geometry optimisation. The lowest-energy arrangement of 2 layers of Sn on Ru has the structure shown in Fig. 2. A second energy minimum was found, in which the second tin layer is identical to the first. The total energy of this structure is only $0.04 \mathrm{eV}$ higher than that of the first. Two key observations can be made: (1) for the lowest-energy structure, the arrangement of the second layer of tin departs significantly from that of the flat underlying layer, forming a staggered, undulating layer; (2) and the energy of the Sn atoms is markedly higher than those of the first layer, resulting in an adsorption energy that is slightly positive: $+0.07 \mathrm{eV}$ relative to bulk $\beta$-Sn. This is a sharp departure from the strongly negative adsorption energy of $-1.19 \mathrm{eV}$ for atoms in the first layer. This implies that while the first layer adsorbs strongly to the underlying Ru surface, the second layer does not bind to the Sn layer beneath, and remains close in energy to the bulk state. The observed slightly positive adsorption energy for the second layer of $\mathrm{Sn}$ atoms indicates that there is no tendency for tin debris on a ruthenium surface to spread into a second smooth layer.

3 \& 4 layers. Both our calculations and previous experiments suggest a tendency of tin towards three-dimensional clustering.

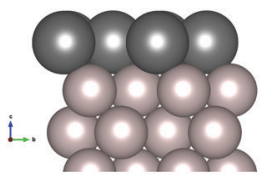

(a)

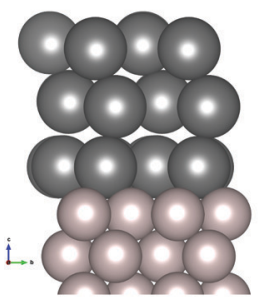

(c)

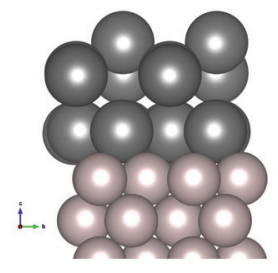

(b)

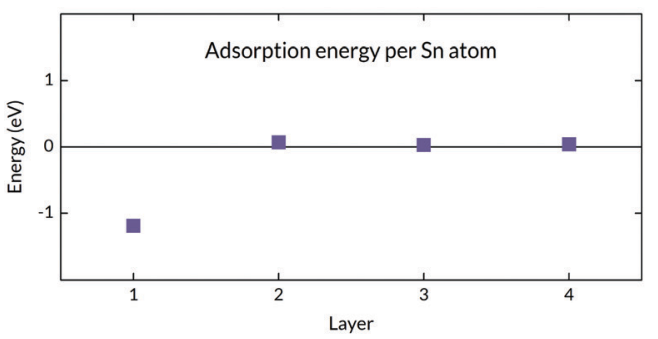

(e)

Fig. 2 (a)-(d) Structures of Sn on Ru, with 1-4 layers (side view; dark spheres represent $\mathrm{Sn}$, light spheres $\mathrm{Ru}$ ), and (e) average energy of adsorption per atom in $\mathrm{Sn}$ layers, relative to $\beta$-tin $(0 \mathrm{eV})$. Note that the adsorption energies are averaged over atoms in each successive layer from (a)-(d), thereby showing the strength of adsorption to the layer immediately beneath.

This means that there will be regions with multiple layers of tin, even when relatively small amounts of tin are deposited on a ruthenium film. We model these regions by adding multiple tin layers in our periodic supercell.

Calculations for a third layer of tin shows that it also forms an uneven sheet of atoms. This behavior extends to 4 layers, the most we have examined. Here too, we find that the energy per atom is slightly higher than the $\beta$-Sn reference. For both the 3 rd and 4 th layers, the adsorption energy is slightly positive, $0.03 \mathrm{eV}$. The observed minimum energy structures and corresponding energies (Fig. 2) indicate that after the layer of tin in direct contact with the ruthenium surface, subsequent layers adopt a structure that is more like that of $\beta$-Sn. One peculiarity is notable: in the 4-layer structure shown, the two bottom layers are identical and flat, with the top two layers taking the aforementioned corrugated form. Considering that the 2-layer structure with identical layers was only $0.04 \mathrm{eV}$ higher than the lowestenergy arrangement, the presence of the 3rd and 4th layers appears to have shifted the energies to favour such a structure.

Sn growth on Ru. The energies and structures reported in the preceding sections prompt a number of conclusions. Sn 
binds strongly to the $\mathrm{Ru}$ surface, with a strongly negative adsorption energy, and with a smooth layer; therefore it confirms the conjecture by Paffet et $a .^{11}$ that the $\mathrm{Sn}-\mathrm{Ru}$ metal-metal bonding enthalpy is greater than that of $\mathrm{Sn}-\mathrm{Sn}$ bonding. This strong bonding is entirely consistent with the finding that $\mathrm{Sn}$ cannot be etched completely from a $\mathrm{Ru}$ substrate. However, subsequent Sn layers do not bind strongly to the Sn underneath. Furthermore, the atoms in the second and higher number of layer(s) are arranged in a distinctly different way (resembling the bulk Sn) from the first layer.

Taken together, these findings indicate that the deposition of tin on ruthenium should follow the so-called StranskiKrastanov growth mode. Here we observe this mode of epitaxial growth in the formation of a single-layer complete tin film on the substrate, after which three-dimensional islands develop. We see in Fig. 2e that there is a strong thermodynamic advantage to the formation of a single flat layer of tin. This advantage is absent for a second layer and subsequent layers, since a tin atom in the bulk, and therefore in 3D islands, is at approximately the same energy level.

\subsection{Sn on $\mathrm{H} / \mathrm{Ru}$}

The main motivation for this study is the blistering of multi-layer mirrors observed when tin debris from the EUV source plasma lands on the ruthenium surface. Hydrogen plasma is used to etch Sn contamination from the surface of the optics. As hydrogen may be present before tin, we consider next the situation when the ruthenium surface is exposed to hydrogen prior to tin exposure.

Hydrogen adsorbs and dissociates readily on the $\mathrm{Ru}(0001)$ surface. ${ }^{8,24}$ All adsorption sites for $\mathrm{H}$ atoms have negative adsorption energies, with a preference for the sites with 3-fold coordination, commonly referred to as the $f c c$ and $h c p$ sites. These have adsorption energies of -0.63 and $-0.57 \mathrm{eV}$ respectively. With $\mathrm{H}$ atoms exhibiting such affinity for the Ru surface, it is to be expected that this will affect the $\mathrm{Sn}-\mathrm{Ru}$ interaction. This indeed proves to be the case. A single Sn atom on the H-covered $\mathrm{Ru}$ (at a concentration of $1 \mathrm{H}$ atom per $\mathrm{Ru}$ atom) has a positive $(+0.21 \mathrm{eV})$ adsorption energy, with $\beta$-Sn as the reference (Fig. 3). For a full Sn layer, the adsorption energy per atom is $0.13 \mathrm{eV}$. We have also performed a comparison of diffusion barriers calculated with the CINEB algorithm, and it shows a large difference in mobility. The potential energy surface for $\mathrm{Sn}$ adsorbed on $\mathrm{Ru}$ is quite flat, ${ }^{8,13}$ and the energy barrier for diffusion from one 3-fold site to the next is at most $0.13 \mathrm{eV}$ on a clean $\mathrm{Ru}$ surface. This changes to $1.0 \mathrm{eV}$ for $\mathrm{Sn}$ on an H-covered surface.

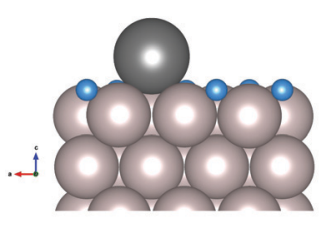

(a)

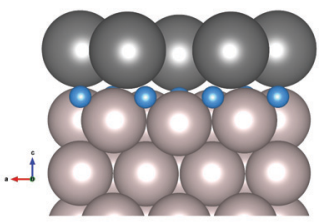

(b)
Fig. 3 Sn on H/Ru(0001); (a) lone Sn atom (b) dense-packed layer of Sn. Dark grey spheres represent $\mathrm{Sn}$, light spheres $\mathrm{Ru}$, bright blue spheres $\mathrm{H}$.
Tin is therefore unlikely to spread and form a single-atom wetting layer on the hydrogen-passivated surface, and should remain in $3 \mathrm{D}$ islands instead. This appears to explain the findings reported by Faradzhev and Sidorkin, ${ }^{25}$ who reported a Volmer-Weber mechanism of $\mathrm{Sn}$ growth on $\mathrm{Ru}$.

\subsection{H on $\mathrm{Sn} / \mathrm{Ru}$}

In previous studies, we examined the effect of adsorbed tin atoms and tin hydrides on the interaction of hydrogen and ruthenium, and found a considerable effect on the penetration of hydrogen into ruthenium. We go further here and calculate the energies for hydrogen on, in and under several layers of tin on the ruthenium. We consider also the implications of the computed energies for a multi-layer mirror exposed to atomic hydrogen, as found in the EUV-induced plasma in the lithographic machines of interest.

1 layer. The adsorption of hydrogen on the tin overlayer is not energetically favourable. A hydrogen atom adsorbed directly on an Sn atom - the top site - has an adsorption energy of $+0.7 \mathrm{eV}$. This can be interpreted to mean that dissociative adsorption of $\mathrm{H}_{2}$ comes at a considerable energy cost, and also that hydrogen atoms on the Sn surface would tend to recombine and desorb from the unfavourable surface.

With only $2 \mathrm{Sn}$ per $3 \mathrm{Ru}$ atoms fitting into the densest packing of the deposited overlayer, the result is a porous Sn layer through which the small $\mathrm{H}$ atom can access the $\mathrm{Ru}$ surface. Fig. 4 shows the computed structures. Although the number of available sites is reduced, $\mathrm{H}$ can adsorb at the favoured sites with 3 -fold coordination, the $f c c$ and $h c p$ sites of the $\mathrm{Ru}(0001)$ surface. The preference for these sites is unchanged relative to pristine $\mathrm{Ru}$, with one key difference: the adsorption energy relative to the $\mathrm{H}_{2}$ is no longer negative ( $h c p$ : $-0.58 \mathrm{eV} ; f c c$ : $-0.63 \mathrm{eV}$ ), but positive ( $h c p:+0.17 \mathrm{eV} ; f c c:+0.18 \mathrm{eV})$.

Although these positive energies would indicate that dissociative adsorption of $\mathrm{H}_{2}$ on $\mathrm{Ru}(0001)$ is hindered by the $\mathrm{Sn}$ layer, atomic hydrogen would favour the Ru surface. The hydrogen radicals in an (EUV-induced) plasma are at a much higher energy level than $\mathrm{H}$ in an $\mathrm{H}_{2}$ molecule (2.27 eV higher), which means that the $\mathrm{Sn} / \mathrm{Ru}$ surface is energetically favourable, and is rapidly populated when atomic hydrogen is supplied.

2 layers. The presence of a second layer of tin hardly changes the energy landscape of a hydrogen atom in the system. As in the case with a single layer, the lowest-energy state is the $\mathrm{H}_{2}$ molecule. Adsorption of $\mathrm{H}$ on the $\mathrm{Sn}$ film remains unfavourable, with a positive adsorption energy for $\mathrm{H}$ on the top site shown in Fig. 4d.

An additional tin layer presents the possibility of an interstitial state, as an $\mathrm{H}$ atom can occupy the subsurface sites between the atoms of the first and second layers of the deposited tin (Fig. 4e). However, interstitial hydrogen in the tin is a relatively high-energy, unfavourable state, $+0.7 \mathrm{eV}$ above the $\mathrm{H}_{2}$ reference. This is to be expected, as the solubility of hydrogen in tin is not high. This state is therefore unlikely to be occupied at any considerable concentration when the $\mathrm{Ru} / \mathrm{Sn}$ is exposed to molecular hydrogen. Here too the Ru surface sites with 3-fold coordination are the lowest in energy for an $\mathrm{H}$ atom. 


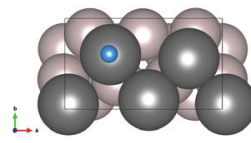

(a) Sn top site

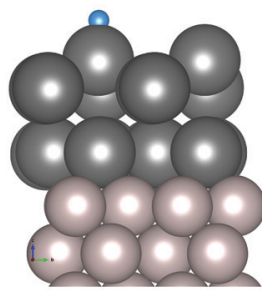

(d) H on top of $\mathrm{Sn}$ overlayer

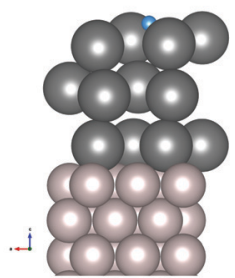

(g) H on top of $\mathrm{Sn}$ overlayer

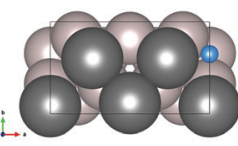

(b) Ru hcp site

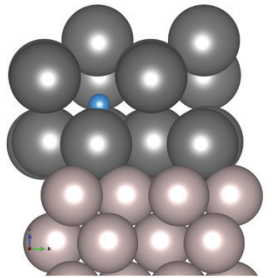

(e) $\mathrm{H}$ between $\mathrm{Sn}$ layers

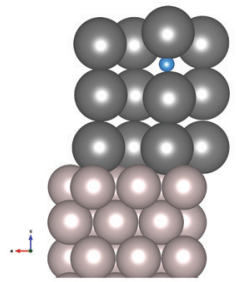

(h) $\mathrm{H}$ under top $\mathrm{Sn}$ layer

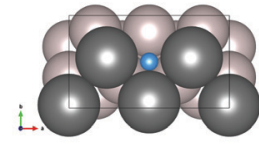

(c) Ru fcc site

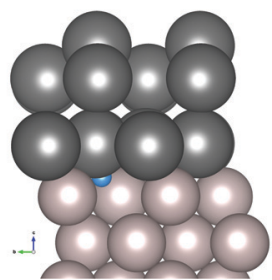

(f) $\mathrm{H}$ at $\mathrm{Sn} / \mathrm{Ru}$ interface, fcc site.

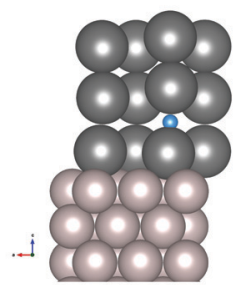

(i) $\mathrm{H}$ on bottom $\mathrm{Sn}$ layer
Fig. 4 (a)-(c) H on 2/3ML Sn/Ru,top view; (d)-(f) H on Sn/Ru 4/3ML, side view; (g)-(i) $\mathrm{H}$ on $2 \mathrm{ML} \mathrm{Sn} / \mathrm{Ru}$, side view. Dark grey spheres represent $\mathrm{Sn}$, light spheres $\mathrm{Ru}$, bright blue spheres $\mathrm{H}$.

However, the adsorption energy becomes yet more positive, now $+0.28 \mathrm{eV}$ for $h c p,+0.31 \mathrm{eV}$ for $f c c$ (Fig. 4f).
The case with atomic hydrogen is of course different, with the hydrogen atom starting at a much higher energy level. All of the afore-mentioned sites on the surface of and between the layers of tin are lower in energy than a hydrogen radical, and the amount of hydrogen in those states would be greater than under only $\mathrm{H}_{2}$.

3 layers. At 3 layers of tin, a general picture emerges for the $\mathrm{H}-\mathrm{Sn}-\mathrm{Ru}$ interactions at the surface of the multi-layer mirror. $\mathrm{H}$ adsorption on the $\mathrm{Sn}$ film is unlikely, due to the positive adsorption energy, $+0.6 \mathrm{eV}$ adsorption energy for the hollow site. Atomic hydrogen should result in increased adsorption of $\mathrm{H}$ on the surface, but with the Sn overlayer present, $\mathrm{H}$ favours the molecular state more than the surface state and the interstitial states, both in the region directly under the surface Sn atoms and deeper.

\subsection{The progress of $\mathrm{H}$ through $\mathrm{Sn}$ and $\mathrm{Ru}$}

Using the CINEB algorithm, we performed transition state searches for hydrogen diffuse pathways between the minima described in the preceding sections. For overall comparison, we consider three key structures: a clean $\mathrm{Ru}(0001)$ surface, $\mathrm{Ru}+2 / 3$ ML of Sn (1 layer), and $\mathrm{Ru}+2 \mathrm{ML}$ of $\mathrm{Sn}$ (3 layers). These are summarised in Fig. 5 .

With no tin on the $\mathrm{Ru}$ surface, the energies of hydrogen indicate a strong preference for the adsorbed, dissociated state. The negative adsorption energy $\left(-0.63 \mathrm{eV}\right.$ relative to the $\mathrm{H}_{2}$ molecule) puts this state far below all the other energy minima encountered in our calculations for $\mathrm{H}, \mathrm{Sn}$, and $\mathrm{Ru}$. Moreover, the energy barrier between the adsorbed state and the subsurface interstitial state is $1.06 \mathrm{eV}$. This indicates a low likelihood of

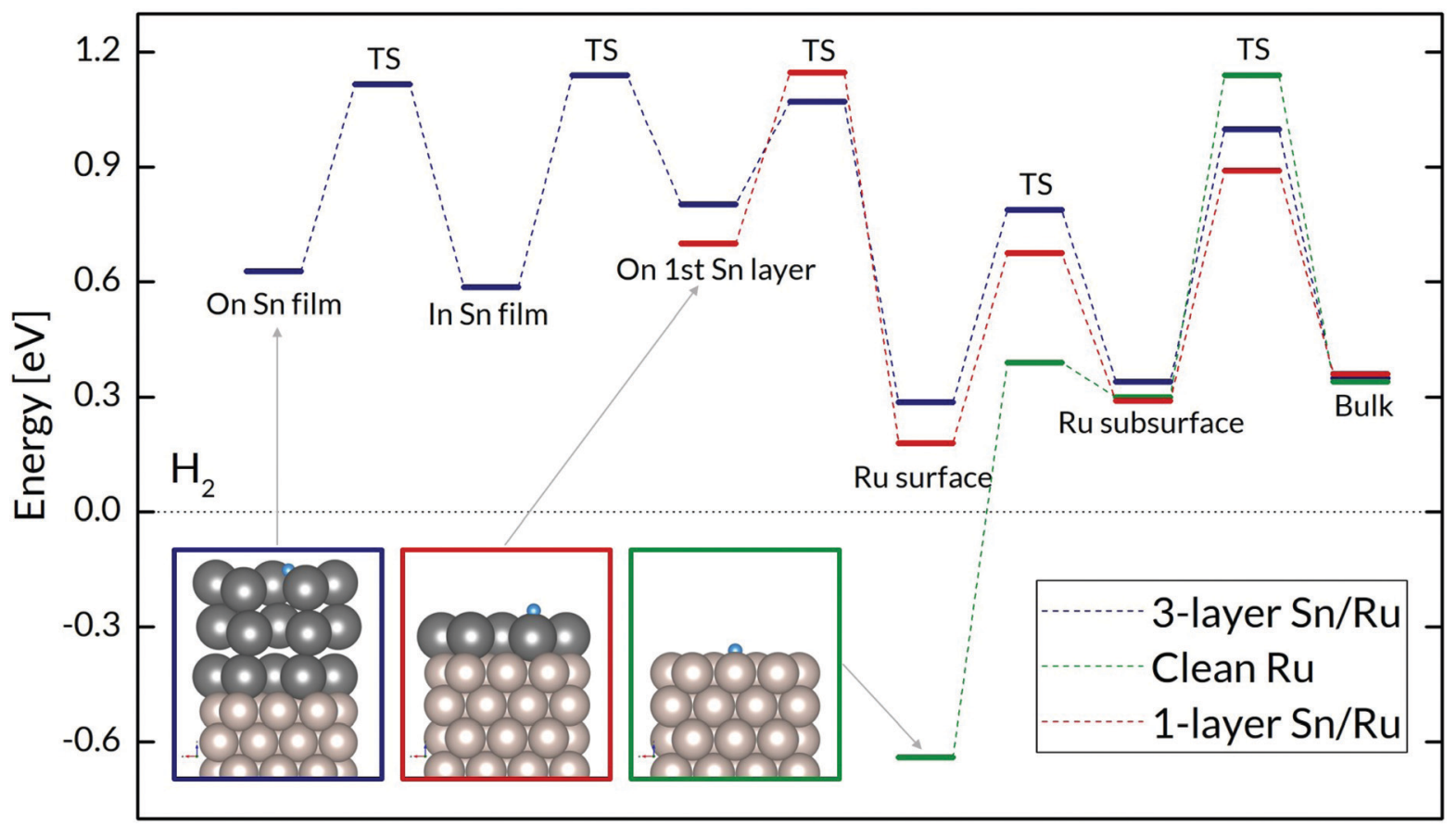

Fig. 5 Energy profile of $\mathrm{H}$ on and in Sn on Ru with no Sn (green), 1 layer of Sn (red), and 3 layers of Sn (blue). The figures in boxes of corresponding colour show the starting point (leftmost marker) of the evaluated path of hydrogen from the surface to the ruthenium bulk. 


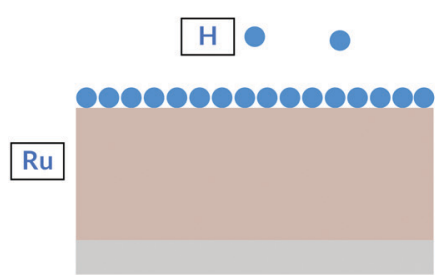

1

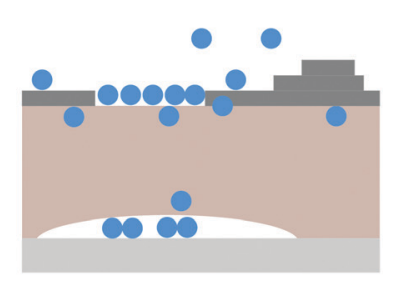

II

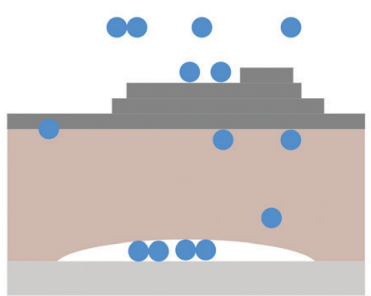

III

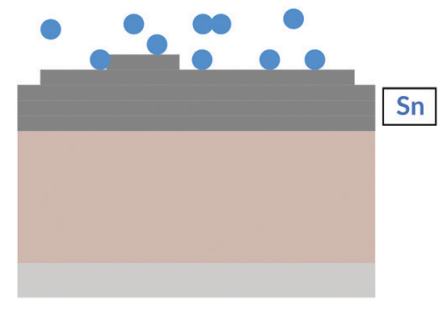

IV

Tin-facilitated blistering

Fig. 6 Four states of tin deposition on ruthenium and their effect on the likelihood of tin-facilitated blistering.

hydrogen permeation through the clean ruthenium. Also Ru has a $+0.34 \mathrm{eV}$ energy of formation for hydride, where hydrogen locates at interstitial site. By all indications, hydrogen concentration in the ruthenium bulk, and at the interface underneath the ruthenium, should remain quite low.

The presence of a Sn overlayer causes two significant changes in the energy landscape. First, the Ru surface site (underneath $\mathrm{Sn}$ ) remains the lowest in energy, although, at $+0.18 \mathrm{eV}$ above the molecular state, it is not as favourable for hydrogen occupation as a clean Ru surface. The second change is in the energy barrier for hydrogen penetration, which falls to $0.5 \mathrm{eV}$. This halving of the energy barrier for hydrogen penetration is accompanied by an increase in the barrier of the reverse process. Thus, with the $\mathrm{Sn}$ present, hydrogen is more likely to go from the Ru surface to the subsurface, and less likely to go from the subsurface to the surface.

The progress of hydrogen through 3 layers of tin entails passing through several high-energy states. First, the adsorption on the top layer is energetically unfavourable, lying $0.6 \mathrm{eV}$ above the $\mathrm{H}_{2}$ molecular state. Then follow the interstitial states within the film, as well as the transition states between them, which are all above the reference state. The energy barriers in the path from the Sn surface to the Ru surface are, respectively in eV: 0.50 , $0.56,0.33$. Compared to the case with only 1 relatively porous layer of tin, the likelihood of an $\mathrm{H}$ atom to travel through to the ruthenium surface is much reduced.

\subsection{Atomic hydrogen and blistering}

The main motivation for this study is the blistering of multilayer mirrors observed when tin debris from the EUV source is deposited on the ruthenium surface. Hydrogen is used to etch Sn contamination from the surface of the optics, and the removal of contamination from the mirror surface with plasma is the subject of numerous publications. The energies reported and discussed in the preceding section are calculated relative to molecular hydrogen, the reference state to which $\mathrm{H}$ from the $\mathrm{Sn} / \mathrm{Ru}$ returns via recombination and desorption. However, EUV-induced plasma is present in the lithographic machines, with the result that atomic hydrogen can make contact with the ruthenium-capped mirrors.

As the $\mathrm{H}$ radicals are at a higher energy level, all the states in Fig. 4 are more accessible. Therefore, with low enough Sn coverage and exposure to hydrogen plasma, a large number of $\mathrm{H}$ atoms will reach the $\mathrm{Ru}$ surface, and thereafter the subsurface. In view of the inhibition of out-diffusion by adsorbates, the right amount of tin will lead to blistering.

The Stranski-Krastanov growth mode implies that further tin deposition onto the first Sn wetting layer will leave part of the Sn overlayer uncovered up to a certain point, after which continued deposition of tin will fill in the "valleys". Working in the opposite direction, etching of Sn layers with $\mathrm{H}$ radicals will likely proceed without blistering, until the last, tightly-bound wetting layer is exposed, at which point $\mathrm{H}$ entry into $\mathrm{Ru}$ is enabled. We can therefore delineate four (4) different states for the $\mathrm{Ru} / \mathrm{Sn} / \mathrm{H}$ system, with differing probabilities of significant $\mathrm{H}$ penetration, and the implications for blistering in each (see Fig. 6):

I pristine $\mathrm{Ru}$ - surface is saturated with dissociated $\mathrm{H}$ atoms, no blistering;

II incomplete Sn overlayer $-\mathrm{H}$ atoms penetrate $\mathrm{Ru}$ through the porous Sn layer and at the edges, in proximity to Sn atoms, blistering;

III complete Sn wetting layer with 3D mounds - $\mathrm{H}$ atoms penetrate Ru through the porous Sn layer, blistering;

IV multiple Sn layers covering Ru surface - Sn layers provide additional obstacles to $\mathrm{H}$ diffusion, no blistering.

\section{Conclusion}

We have carried out a computational study of the deposition of tin on the ruthenium surface, through calculation of the structures and energies of various amounts of tin, from one layer to several. The calculations have shown that tin will spread to form a single-layer film on the ruthenium, and thereafter grow by forming three-dimensional islands. We show also that tin does not adsorb strongly on a hydrogen-covered ruthenium surface. We have performed transition state calculations for the hydrogen diffusion paths through a tin film consisting from one to several layers to the ruthenium surface and subsurface. The results show that multiple layers of Sn will obstruct the hydrogen atoms, whereas a single or incomplete layer will allow and facilitate hydrogen permeation. 


\section{Conflicts of interest}

There are no conflicts of interest to declare.

\section{Acknowledgements}

This research was carried out under project number T16010a in the framework of the Partnership Program of the Materials innovation institute M2i (www.m2i.nl) and the Technology Foundation TTW (www.stw.nl), which is part of the Netherlands Organization for Scientific Research (www.nwo.nl).

\section{Notes and references}

1 Y. B. He, A. Goriachko, C. Korte, A. Farkas, G. Mellau, P. Dudin, L. Gregoratti, A. Barinov, M. Kiskinova, A. Stierle, N. Kasper, S. Bajt and H. Over, J. Phys. Chem. C, 2007, 111, 10988-10992.

2 S. Bajt, H. N. Chapman, N. Nguyen, J. B. Alameda, J. C. Robinson, M. E. Malinowski, E. Gullikson, A. Aquila, C. Tarrio and S. Grantham, Proc. SPIE, 2003, 5037, 236-248.

3 M. M. J. W. van Herpen, D. J. W. Klunder, W. A. Soer, R. Moors and V. Banine, Chem. Phys. Lett., 2010, 484, 197-199.

4 D. T. Elg, G. A. Panici, S. N. Srivastava and D. N. Ruzic, Extreme Ultraviolet (EUV) Lithography VI, 2015, 9422, 94222H.

5 D. Qerimi, G. Panici, A. Jain, D. Jacobson and D. N. Ruzic, J. Vac. Sci. Technol., B: Nanotechnol. Microelectron.: Mater., Process., Meas., Phenom., 2020, 38, 052601.

6 A. S. Kuznetsov, R. W. E. van de Kruijs, M. A. Gleeson, K. Schmid and F. Bijkerk, J. Surf. Invest.: X-Ray, Synchrotron Neutron Tech., 2010, 4, 563-566.

7 A. S. Kuznetsov, M. A. Gleeson and F. Bijkerk, J. Appl. Phys., 2014, 115, 173510.

8 C. Onwudinanti, I. Tranca, T. Morgan and S. Tao, Nanomaterials, 2019, 9, 1-18.
9 C. Onwudinanti, G. Brocks, V. Koelman, T. Morgan and S. Tao, Phys. Chem. Chem. Phys., 2020, 22, 7935-7941.

10 B. N. Popov, J.-W. Lee and M. B. Djukic, Handbook of Environmental Degradation of Materials, Elsevier, 3rd edn, 2018, pp. 133-162.

11 M. T. Paffett, A. D. Logan and T. N. Taylor, J. Phys. Chem., 1993, 97, 690-695.

12 J. Yuhara, Y. Ishikawa and T. Matsui, Surf. Sci., 2013, 616, 131-136.

13 R. Topolnicki and R. Kucharczyk, Appl. Surf. Sci., 2015, 329, 376-383.

14 M. Pachecka, J. M. Sturm, R. W. E. van de Kruijs, C. J. Lee and F. Bijkerk, AIP Adv., 2016, 6, 075222.

15 D. T. Elg, J. R. Sporre, G. A. Panici, S. N. Srivastava and D. N. Ruzic, J. Vac. Sci. Technol., A, 2016, 34, 021305.

16 D. T. Elg, G. A. Panici, S. N. Srivastava and D. N. Ruzic, Extreme Ultraviolet (EUV) Lithography VII, 2016, 9776, 97760M.

17 G. Kresse and J. Hafner, Phys. Rev. B: Condens. Matter Mater. Phys., 1994, 49, 14251-14269.

18 G. Kresse and J. Furthmüller, Comput. Mater. Sci., 1996, 6, 15-50.

19 D. Joubert, Phys. Rev. B: Condens. Matter Mater. Phys., 1999, 59, 1758-1775.

20 J. D. Perdew, K. Burke and M. Ernzerhof, Phys. Rev. Lett., 1996, 77, 3865-3868.

21 G. Henkelman, B. P. Uberuaga and H. Jónsson, J. Chem. Phys., 2000, 113, 9901-9904.

22 H. King, CRC Handbook of Chemistry and Physics, CRC Press, 2012, pp. 15-18.

23 K. K. Irikura, J. Phys. Chem. Ref. Data, 2007, 36, 389-397.

24 I. Del Rosal, L. Truflandier, R. Poteau and I. C. Gerber, J. Phys. Chem. C, 2011, 115, 2169-2178.

25 N. Faradzhev and V. Sidorkin, J. Vac. Sci. Technol., A, 2009, 27, 306-314. 